\title{
Magensäureblocker - des Guten zu viel?
}

\begin{abstract}
H2-Rezeptoren-Antagonisten und vor allem die Protonenpumpeninhibitoren (PPI) haben die Behandlung von peptischen Ulzera und Refluxkrankheit revolutioniert. Sie zählen zu den weltweit am meisten verordneten Medikamenten und rangieren mit einem globalen Umsatz von 26,5 Milliarden US-\$ (2008) auf Platz 5 der umsatzstärksten Medikamentengruppen. Mehr Geld ausgegeben wird nur für Krebsmittel, Lipidsenker, Asthma-/COPD-Medikamente und Antidiabetika. Eine große Anzahl von Patienten jedoch - Studien sprechen von bis zu 90\% - nehmen diese Magensäureblocker ohne adäquate Indikation ein.
\end{abstract}

— Obwohl sie insgesamt gut verträglich sind, ist ihr Nebenwirkungspotenzial vor allem bei älteren Menschen ernster als bisher angenommen. So werden Pneumonien unter PPI-Therapie gehäuft beobachtet, und zwar sowohl Krankenhauspneumonien als auch ambulant erworbene Fälle von Lungenentzündung. Auch das mehr als dreimal häufigere Auftreten von Enteritiden durch Salmonella und Campylobacter wird mit dem Verlust der natürlichen Säurebarriere erklärt. Die Suppression der Säureproduktion erleichtert die Kolonisation des normalerweise sterilen oberen Gastrointestinaltraktes, Bakterien aus tieferen Darmabschnitten können sich nach oral ausbreiten und per os aufgenommene enteropathogene Keime erreichen tiefere Regionen, wo sie Enteritiden verursachen.

Ein weiterer gravierender Befund ist die Verdoppelung von Clostridium-difficile-assoziierten Erkrankungen unter PPI. Patienten, die wegen Clostridiumdifficile-assoziierten Erkrankungen behandelt werden, haben ein höheres Rezidivrisiko, wenn sie weiterhin PPI erhalten. Darüber hinaus gibt es Hinweise auf die Entstehung einer Hypomagnesiämie durch Hemmung der intestinalen Magnesiumresorption unter Behandlung mit Protonenpumpeninhibitoren, und auch eine Eisen- und Vitamin-B12-Malabsorption kommt offenbar häufiger vor. Weiterhin gibt es in jüngerer Zeit Hinweise auf einen $\mathrm{Zu}$ sammenhang von PPI mit gehäuft auftretenden osteoporotischen Frakturen.

Die Autoren folgern [1], dass durch die inadäquate Verordnung von PPI erhebliche Ressourcen unnütz vergeudet werden und dass der unkritische Einsatz

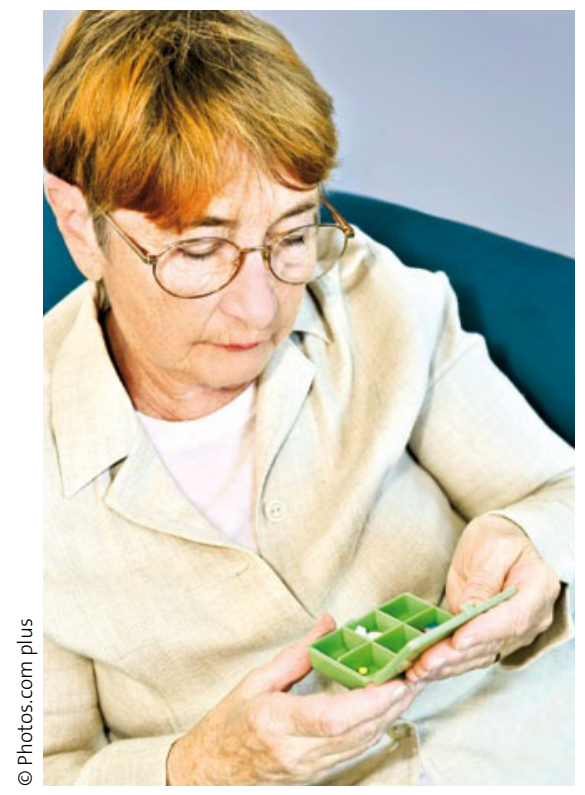

PPI werden älteren Patienten zu oft ohne ausreichende Indikation verordnet.

von PPI eine Zunahme der Pneumoniehäufigkeit und vermehrte Clostridium-difficile-Erkrankungen nach sich zieht. Darüber hinaus gibt es Hinweise auf weitere ungünstige Auswirkungen von PPI auf die Gesundheit vor allem älterer Menschen (Hypomagnesiämie, osteoporotische Knochenbrüche, Eisenund Vitaminmangel).

\section{Kommentar}

Die Hinweise auf schwere unerwünschte Nebenwirkungen von Protonenpumpeninhibitoren („Prazole“), vor allem ältere Menschen betreffend, mehren sich. Die Autoren der vorgestellten Studien rechnen vor, dass bis zu 0,9\% aller im Krankenhaus erworbenen Pneumonien den PPI anzulasten sein und damit bis zu 33000 Todesfälle pro Jahr allein in den USA vermieden werden könnten [2]. Auch vermehrt auftretende Enteritiden und clostridienbedingte Erkrankungen werden zunehmend mit PPI in Verbindung gebracht. Andererseits haben PPI einen wichtigen und etablierten Stellenwert bei der Behandlung peptischer Erkrankungen. Um gezielter behandeln zu können und eine unnötige oder sogar gefährliche Gabe von PPI zu vermeiden, fragen wir bei unseren geriatrischen Patienten, die bei Krankenhausaufnahme sehr häufig PPI in ihrer Medikamentenliste stehen haben, gezielt nach:

(1) Anamnese von Ulcera duodeni und UIcera ventriculi sowie Helicobacter pylori, (2) oberen GI-Blutungen in den letzten Jahren,

(3) Einnahme von NSAR (ggf. in Kombination mit ASS, Kortikosteroiden oder oralen Antikoagulanzien),

(4) Refluxkrankheit bzw. Sodbrennen. Bei Patienten, die eine oder mehrere dieser Fragen positiv beantworten, sind wir beim Absetzen der PPI vorsichtig, gelegentlich liefert eine Gastroskopie Entscheidungshilfe. In Übereinstimmung mit der Literatur findet sich jedoch bei zwei Drittel bis drei Viertel der PPI-behandelten Patienten keine hinreichende Indikation zur Säurehemmung. Ein kontrollierter und im Arztbrief an den Hausarzt erwähnter Absetzversuch verläuft bei solchen Patienten zu einem hohen Anteil erfolgreich. Wichtig dabei ist, dass das probatorische Absetzen ausschleichend über ca. vier Wochen erfolgen sollte.

Durch eine gezieltere und kritischere Verordnungsweise können zum einen potenziell gravierende PPI-Folgeerkrankungen (Pneumonien, Enteritiden) vermieden und zum anderen sehr erhebliche Mittel sinnvoll gespart werden.

J. ZeeH -

1. I. C. Logan, D. Sumukadas, M. D. Witham Gastric acid suppressants - too much of a good thing? Age and Ageing 39 (2010) 410-411

2. S. J. Herzig et al.

Acid-suppressive medication use and the risk for hospital acquired pneumonia. J. Am. Med. Ass. 301 (2009) 2120-2128 